\title{
Displaced Peritoneal Dialysis Catheter
}

National Cancer Institute

\section{Source}

National Cancer Institute. Displaced Peritoneal Dialysis Catheter. NCI Thesaurus. Code C115162.

Malposition and dysfunction of a peritoneal dialysis catheter. 